\title{
Sunscreens and photoprotection
}

\section{Marco Andreassi}

To cite this article: Marco Andreassi (2011) Sunscreens and photoprotection, Expert Review of Dermatology, 6:5, 433-435, DOI: 10.1586/edm.11.59

To link to this article: https://doi.org/10.1586/edm.11.59

Published online: 10 Jan 2014.

Submit your article to this journal 4

Џ Article views: 800

Citing articles: 4 View citing articles 다 


\section{Sunscreens and photoprotection}

Expert Rev. Dermatol. 6(5), 433-435 (2011)

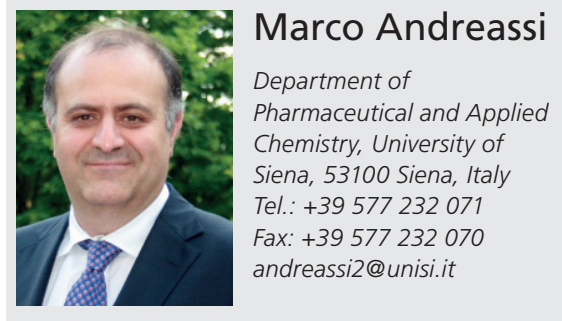

EXPERT
REVIEWS

\section{“This special focus issue on 'Sunscreens and photoprotection' highlights recent advances in the technology of products, and also provides an update on the main dermatological pathologies that could be prevented with photoprotection."}

Skin cancer incidence is continuously increasing, and sun exposure has been indicated as the primary cause of such an explosion in prevalence. The phenomenon is particularly pronounced among Caucasians living in developed countries, suggesting that some changes in lifestyle may be the main trigger. Generally, most people consider suntan as a sign of physical and social wellbeing, and the desire to acquire a suntan has promoted holidays in sunny countries, also out of season. Furthermore, the use of artificial tanning devices that emit UVA/UVB radiation has become increasingly popular among young people, especially females.

In this scenario a change of direction can be obtained by education on the correct way to enjoy sun exposure from childhood, but this requires a large organizational effort, which is difficult to achieve in a short time. In the immediate future, the implementation of cutaneous photoprotection appears more feasible. In fact, the large-scale use of products able to neutralize the UV radiation has been advocated by many international institutions as the most appropriate means to reduce skin damage from solar radiation, namely photoaging and photocarcinogenesis.

The photoprotection products have to provide evidence of protective ability against solar radiation and, simultaneously, given their wide use, must be toxicologically safe. Both these requirements are encompassed by the current laws that provide lists of permitted substances.
Achieving these goals has required, and still requires, great effort in identifying suitable ingredients and technologically advanced and safe formulations.

This special focus issue on 'Sunscreens and photoprotection' highlights recent advances in the technology of products, and also provides an update on the main dermatological pathologies that could be prevented with photoprotection.

The issue begins with an editorial by Friedman et al. on the emerging role of nanotechnology in sunscreen [1]. Nanotechnology is a topic of primary interest, particularly for inorganic ingredients, zinc oxide and titanium dioxide - today, mainly used as nanoparticles - which have chemical, mechanical, electrical and optical properties substantially different from microparticles.

The second article is a review by Andreassi, concerning UV exposure as a risk factor for skin cancer [2]. Epithelial skin tumors, particularly squamous cell carcinomas (SCCs), are associated with cumulative UV dose, whereas melanoma (MM) appears related to intermittent sun exposure. This seems to be confirmed by experimentally UV-induced MM, suggesting that sunburn in infancy may be an important risk factor for MM. Biomolecular studies have shown that UV radiation promotes the formation of photoproducts able to provoke UV-induced mutations, which have been found with high frequency in SCCs. The role of UV radiation as a risk factor for MM should also be considered in relation to genetic predisposition. In subjects belonging to families with a high 
incidence of $\mathrm{MM}$, genetic alterations, such as variants of the $M C 1 R$ and $C D K N 2 A$ gene mutations, associated with red hair and freckles, have been identified. UV radiation can affect the immune system favoring the development of UV-induced skin cancer.

Sun exposure induces a broad range of skin reactions, the so-called photodermatoses. This subject has been discussed by Calzavara-Pinton et al. in an article on photoprotection and abnormal cutaneous photosensitivity [3]. The authors emphasize that the clinical diagnosis of photodermatoses may be difficult if skin lesions heal spontaneously before medical examination and careful phototesting is needed. The management of the photosensitive patient includes photoprotection, preventive phototherapy and medical treatment of acute flares. The suitable sunscreens should match the individual action spectrum of the disease and it should have a filtering activity proportional to the light threshold. Oral and topical antioxidants are often used as well, but further studies are needed to assess their efficacy.

Subjects of great interest have been discussed by Pacifico et al. in the article 'Photoprotection issues' [4]. The authors have focused on two topical issues: the importance of genetic alterations of the $p 53$ gene and a new approach to photoprotection - that is, the use of repair enzymes incorporated into liposomes. On the first point, the authors stress the importance of the $p 53$ gene as a regulator of the genome and on its role, when mutated, in promoting the growth of keratinocytes that acquire resistance to apoptosis and cell death. These keratinocytes can undergo clonal expansion and promote the onset of actinic keratoses and carcinomas. The second subject concerns the possibility of using purified DNA repair enzymes, and employment of this extract into cosmetic suncare products.

The spectroscopic measurements of sunscreen protection is a subject discussed by Stanfield [5]. This topic is of extreme practical importance, because it would be highly desirable to replace measurements of sun protection factor (SPF) on human subjects with in vitro spectroscopic measurements on artificial substrates. Spectroscopic measurements of SPF would enable the use of UV radiation spectra that accurately simulate sunlight to determine the SPF. Although consistent prediction of SPF using in vitro methods has eluded researchers for more than three decades, it is possible to measure in vitro absorbance spectra of sunscreen formulas and derive absorbance ratios of selected spectral ranges that are consistent with sun protection measurements on human volunteers. Given the wide variety of sunscreen formulas and the complexity of modeling their behavior, it could be productive to perform spectroscopic measurements using a dynamic model and substrates that provide a distribution of film thicknesses simulating those of sunscreens on human skin. This dynamic model may be extended to discrete wavelengths, and used to compute the absorbance spectrum that represents the cumulative behavior of the sunscreen during irradiation with a UV dose in minimal erythemal doses corresponding to the SPF. That spectrum, known as the integrated absorbance spectrum, is the appropriate spectrum for use in computing the critical wavelength and other absorbance ratios.

The review by Osterwalder et al. focuses on the advance in sunscreens to prevent skin cancer [6]. Indeed, aside from reducing sunburn, modern-day sunscreens also have the potential to prevent skin cancer and slow photoaging, if used appropriately. The article traces through the evolution of sunscreens and highlights the clinical benefits, specifically in preventing skin cancers. Besides improved UV filter technology, development of UVA assessment methods and the regulatory standards are instrumental in the advancement of sunscreen efficacy. Lastly, compliance remains the most crucial factor for optimal sun protection. Sunscreen is often applied in too little amounts, too late or not at all. Compliance may not improve if users do not change their behaviors and habits.

The article by Andreassi et al. emphasizes some hot topics on UV filter ingredients that are used in sunscreens, which are regulated by laws and lists, constantly updated on the basis of scientific information [7]. Most advanced countries have regulatory bodies that publish and update the list of authorized substances. Inorganic filters, zinc oxide and titanium dioxide are often used as nanostructured forms. Nanoparticles have been the subject of technological studies aimed at improving efficacy and safety. The organic ingredients are able to absorb UV radiation on a selective or a broad spectrum. The latter are preferable given the role of UVA radiation in skin carcinogenesis. The organic filters may interact with epidermal proteins and induce sensitization. They can also be absorbed causing systemic effects.

Lipid nanoparticles as nanocarriers for more effective and safer photoprotective products is a topic of great relevance, that is discussed by Nikolić et al. [8]. Lipid nanoparticles possess a solid particle matrix ideal for the incorporation of lipophilic sunscreens. Incorporation of the sunscreens into the nanoparticles increases their UV protective effect compared with oil-in-water emulsions. Due to the solid state of the particle matrix, the release of suncreens is lower compared with emulsions, and thus the sunscreens remain longer on the skin penetration into the skin and related side effects are reduced. Small-sized particulate UV protectants (e.g., $15 \mathrm{~nm}$ ), such as titanium dioxide, are being tested/developed; however, these bear the risk of penetrating the skin and therefore may cause side effects. These particulates can also be incorporated into lipid nanoparticles (e.g., $300 \mathrm{~nm}$ ); firm enclosure inside the nanoparticles excludes penetration into the skin, and simultaneously increases UV protection. Lipid nanoparticles can be produced on an industrial scale by high-pressure homogenization; all excipients are regulatorily accepted.

The value of the innovative approach of novel particulate carriers for sunscreen formulations is the subject of the article by Blasi et al. [9]. Today, sunscreen products are based on conventional formulations, such as creams, milks or gels, which do not protect chemical UV filters from photodegradation and allow its systemic absorption. In this article, innovative systems, namely clays, cyclodextrins, polymer and lipid microparticles, are discussed as potential sunscreen carriers to be embedded in the aforementioned formulations. Their capability to improve sunscreen performances, to reduce their potential toxicity issues, and to overcome formulation problems are examined as well.

From its appearance through to the present day, the level of photoprotection offered has achieved great progress, especially in the search for ingredients able to counteract the damage caused by 
UV radiation and in the technological evolution of formulations. The contributions that appear in this issue have thoroughly examined the most important and current aspects of photoprotection, as well as related clinical problems.

Further details, mainly on sunscreen regulations, can be found in [101] and [102], which I would recommend to readers for any additional information on the subject.

\section{Financial \& competing interests disclosure}

The author has no relevant affiliations or financial involvement with any organization or entity with a financial interest in or financial conflict with the subject matter or materials discussed in the manuscript. This includes employment, consultancies, honoraria, stock ownership or options, expert testimony, grants or patents received or pending, or royalties.

No writing assistance was utilized in the production of this manuscript.

\section{References}

1 Nasi A, Wang S, Friedman A. The emerging role of nanotechnology in sunscreens: an update. Expert Rev. Dermatol. 6(5), 437-439 (2011).

2 Andreassi L. UV exposure as a risk factor for skin cancer. Expert Rev. Dermatol. 6(5), 445-454 (2011).

3 Calzavara-Pinton P, Rossi M, Sala M, Venturini M. Photoprotection and abnormal cutaneous photosensitivity. Expert Rev. Dermatol. 6(5), 455-464 (2011).

4 Pacifico A, Leone G, Picardo M. Photoprotection issues. Expert Rev. Dermatol. 6(5), 465-473 (2011).

5 Stanfield JW. Spectroscopic measurements of sunscreen protection. Expert Rev. Dermatol. 6(5), 475-478 (2011).
6 Osterwalder U, Herzog B, Wang SQ. Advance in sunscreens to prevent skin cancer. Expert Rev. Dermatol. 6(5), 479-491 (2011).

7 Andreassi M, Anselmi C. Hot topics on UV filter ingredients. Expert Rev. Dermatol. 6(5), 493-499 (2011).

8 Nikolić S, Gohla S, Müller RH. Lipid nanoparticles: nanocarriers for more effective and safer photoprotective products. Expert Rev. Dermatol. 6(5), 501-507 (2011).

9 Blasi P, Schoubben A, Giovagnoli S, Rossi C, Ricci M. The real value of novel particulate carriers for sunscreen formulation. Expert Rev. Dermatol. 6(5), 509-517 (2011).

\section{Websites}

101 Rulemaking History for OTC Sunscreen Drug Products www.fda.gov/Drugs/

Development ApprovalProcess/ DevelopmentResources/Over-theCounterOTCDrugs/ StatusofOTCRulemakings/ucm072134.htm

102 EU Consumer Affairs - Cosmetics Sunscreen products http://ec.europa.eu/consumers/sectors/ cosmetics/cosmetic-products/sunscreenproducts/index_en.htm 